\title{
Shoulder hemiarthroplasty for fractures of the proximal humerus
}

\author{
R. Castricini $\cdot$ M. De Benedetto $\cdot$ P. Pirani $\cdot$ \\ N. Panfoli $\cdot$ N. Pace
}

Published online: 19 April 2011

(C) The Author(s) 2011. This article is published with open access at Springerlink.com

\begin{abstract}
Proximal humeral fractures were managed with primary hemiarthroplasty in 57 patients, 53 women $(93 \%)$ and 4 men (7\%) aged 51-87 years (mean 72.2). The mean follow-up period was 52 months (range 12-98), and the mean Constant score was 59.2 (range 38-76). Patients were very satisfied $(n=19)$; satisfied $(n=32)$ or dissatisfied with the outcome $(n=5)$. One patient required early revision surgery. Surgical treatment of three- and four-part fractures of the proximal humerus with hemiarthroplasty is a safe and effective approach, the outcome of which appears to be related to the quality of the anatomical reconstruction of the tuberosities.
\end{abstract}

Keywords Hemiarthroplasty - Proximal humeral fracture $\cdot$ Shoulder fractures $\cdot$ Humeral prosthesis

\section{Introduction}

Proximal humeral fractures are the third most frequent limb fracture type in elderly patients. In a Swedish study of 2,125 fractures, Bengner et al. [1] found a significant, gradual increase in their incidence. According to Lind et al. [2], a major contributing factor is the rising proportion of elderly people in the population. They are largely due to osteoporosis of the humerus, and, like fractures of the

R. Castricini $(\bowtie) \cdot$ N. Panfoli $\cdot$ N. Pace

U.O. di Ortopedia e Traumatologia, ASUR Marche, Ospedale

Civile, Jesi, Italy

e-mail: robertocastricini@tin.it

M. De Benedetto · P. Pirani

U.O. di Ortopedia e Traumatologia, Maria Cecilia Hospital-

GVM Care and Research, Cotignola, Ravenna, Italy femoral neck, they are age- and sex-specific, increasing twice as fast in women as in men [3].

Three- and four-part fractures account for 13-16\% of the fractures of the proximal humeral epiphysis [3, 4], and approximately $20 \%$ require surgical management [3]. The surgical approach to three- and four-part fractures is debated. Options include closed reduction and percutaneous pinning with or without isolated screws, open reduction and internal fixation (ORIF) with sutures, alone or combined with hardware, and plating. Fixed-angle plates have been introduced to provide a durable reduction, especially when there is concern over the quality of the bone [5].

The aim of any operative intervention is to preserve the vascularity of the humeral head, avoiding avascular necrosis [6-8]. Most of the humeral head is supplied by the anterolateral branch of the anterior humeral circumflex artery, which is commonly affected in four-fragment fractures. Although not all cases of avascular necrosis progress to collapse of the humeral head, the condition is associated with a significantly worse outcome [6].

Shoulder hemiarthroplasty (HA) is indicated in patients with displaced and comminute fractures, where avascular necrosis of the humeral head seems inevitable. According to Hertel et al. [9], the predictors of humeral head ischaemia are integrity of the medial hinge, length of the dorsomedial metaphyseal extension of the head fracture (calcar length) and fracture type. In the elderly, most displaced three- and four-part fractures, fracture-dislocations and fractures with a split or impacted humeral head with loss of greater than $40 \%$ of the articular surface can be managed by HA [10-13]. In younger individuals, if osteosynthesis cannot provide a stable anatomically reduced proximal humerus, replacement with a prosthetic head may be considered. However, the ability of HA to restore normal shoulder kinematics and function remains a matter of 
controversy [14]. Neer's very good results [15] have not been replicated by all surgeons, thus contributing to the debate between advocates and opponents of shoulder HA for the treatment of these fractures.

We describe the mid term results of primary HA performed to manage three- and four-part fractures of the proximal humerus.

\section{Materials and methods}

Between January 2000 and December 2008, 90 fractures of the proximal humeral epiphysis were managed with HA by one surgeon at Jesi Hospital (Italy). Fractures were classified according to Neer [15] based on preoperative X-rays and intra-operative results, to minimize intra- and interobserver variability. Six different prosthetic devices were used. To reduce the number of variables, this study included only two implants: Aequalis-Tornier $(n=41)$ and TESS-Biomet $(n=25)$. Of these 66 patients, 4 died and 5 were lost to follow-up, leaving 57 patients. There were 7 (12\%) three-part fractures, 42 (73\%) four-part fractures and $8(14 \%)$ fracture-dislocations, accounting for 34 Aequalis and 23 TESS prostheses. All fractures were operated on within 10 days (mean: 3 days). Women were the vast majority of patients $(n=53: 93 \%)$. Mean age at the time of injury was 72.2 years (range: $51-87$ ). The right arm was involved in $24(42.8 \%)$ and the dominant side in $27(48 \%)$ patients. The mechanism of injury was a fall at home in $89 \%$, and a road traffic accident in $11 \%$ of patients.

Statistical analysis was performed by standard methods. A value of $P<0.05$ was considered significant. The test used and the level of significance are reported for each finding.

The operation was performed under general anaesthesia. The patient was placed in the beach-chair position on the edge of the table, with the arm being operated on hanging over the edge. This allowed full mobility of the limb. A deltopectoral approach was adopted in all patients, without detaching the anterior deltoid and the upper third of the pectoralis major. The long head of the biceps was used as a landmark to localize the tuberosities. The fracture line was slightly posterior to the bicipital groove in about $80 \%$ of cases. The humeral head was removed and its diameter measured. In patients with a three-part fracture, the lesser tuberosity, still attached to the head, was resected and the humeral head removed without detaching the subscapularis tendon. After isolation of the tuberosities, non-absorbable sutures were placed at the bone-tendon junction. All four patients with a glenoid fracture had involvement of the antero-inferior border of the articular surface; of these, 2 required screw fixation and in the remaining 2 , the size of the bone fragment was negligible. The humeral canal was then reamed, and a trial stem was inserted to determine height and version (retroversion about $20^{\circ}$ ); in this phase, the Aequalis device requires an extramedullary guide for correct component positioning, using the preoperative $\mathrm{X}$-rays of the contralateral arm as reference. The tuberosities were reduced, and the position and height of the implant checked by fluoroscopy. Stems were cemented, sparing the epiphysis to avoid affecting bone repair. A bone graft from the humeral head was placed between the tuberosities to restore humeral offset. The TESS Corolla was filled with spongy bone from the humeral head added with autologous growth factors (Fig. 1). The tuberosities were then sutured to one another and to the humeral shaft with non-absorbable horizontal and vertical sutures.

In the postoperative period, the arm was placed in an immobilizer in $15^{\circ}$ of abduction for 4 weeks. Passive mobilization and pendulum exercises were allowed immediately. Active mobilization was begun on the 5th week and strengthening at 8 weeks, initially only with isometric exercises and later with elastic bands.

Clinical and radiographic follow-up data were available for 56 patients. Mean follow-up duration was 52.7 months (range 12-98). Outcomes were assessed using the Constant-Murley score, which attributes 20 points for function (ADL), 15 for pain (subjective components), 40 for range of motion and 25 for strength (objective components); the highest total score is 100 , indicating a healthy, asymptomatic joint; and the lowest is 0 [16].

Patients' satisfaction was graded as very satisfied, satisfied and dissatisfied. The X-ray examination included true $\mathrm{AP}$, and, where possible, axial views. At each follow-up visit, all previous radiograms were examined for periprosthetic changes. The acromio-humeral distance (AHD), i.e. the distance between the acromion and the top of the prosthetic head, was always determined, since an AHD less

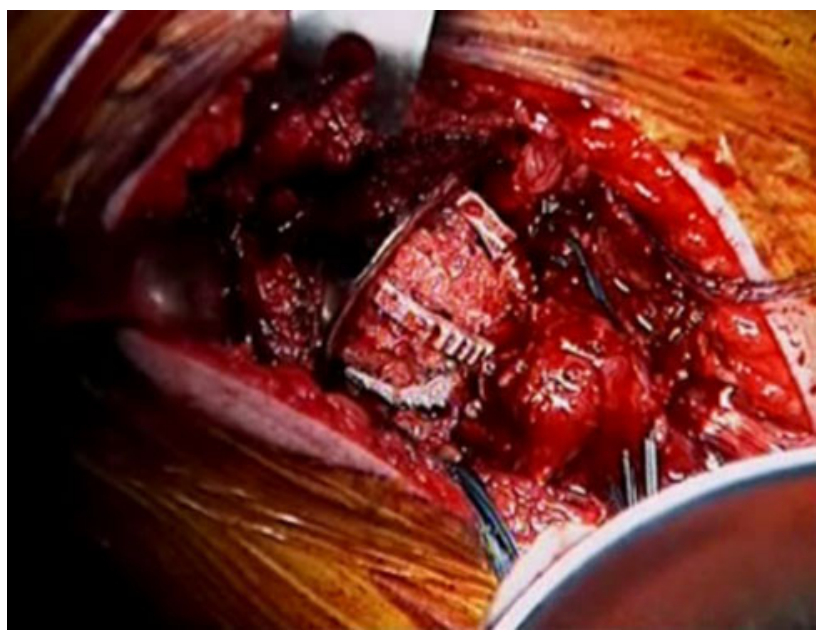

Fig. 1 TESS corolla filled with spongy bone from the humeral head added with autologous growth factors 
than $7 \mathrm{~mm}$ reflects proximal humeral migration. The position and state of the tuberosities were carefully examined. The greater tuberosity was considered to be properly positioned when its top stood 5-10 $\mathrm{mm}$ below the top of the head component. Tuberosity restoration was graded as anatomical reconstruction, malunion $(>0.5 \mathrm{~cm}$ from the head component) and resorption/non-union. Any heterotopic ossifications and the state of cement were recorded.

\section{Results}

The mean Constant score was 59.2 (range 38-76) (Table 1); 19 patients were very satisfied with the outcome of the operation, 32 were satisfied, and 5 were dissatisfied. One patient required early revision surgery with a reverse prosthesis due to stem loosening.

Pain is the main factor in patient satisfaction. The mean pain score was 14/15, with no pain in 45 patients (80\%) and slight, sporadic pain in the other 11 patients; 27 (82\%) Aequalis patients had no pain as opposed to 18 (78\%) TESS patients, whereas $6(18 \%)$ and 5 patients $(22 \%)$, respectively, had sporadic pain (chi-square test, $p r 0.742$ ).

The mean motion score was 25.3/40 (range 10-38); active anterior elevation was greater than $150^{\circ}$ in 12 patients, between 120 and $150^{\circ}$ in 20 (mean $106^{\circ}$ ) and less than $120^{\circ}$ in the other 24 ; mean external rotation was $19^{\circ}$ (range $0-40$ ), and internal rotation was prevalently at the level of L3. The mean ADL score was 16.3 (range 12-20); this is an excellent outcome given the low functional demands of these patients, who are typically quite elderly. The most frequent impairments involved lifting weights, raising the arm above the shoulder, combing one's hair and sleeping on the affected side. The strength score was only $3.3 / 25$ (range $0-8$ ) and was higher in the TESS group (3.7 vs. $3 ; t$ test, $p r$ 0.1505).

The Constant score of the two groups (Aequalis 59.3, TESS 59.1) was not significantly different (Kruskal-Wallis test constant $p r=0.9335$ ).

An AHD less than $7 \mathrm{~mm}$, reflecting insufficiency or rupture of the rotator cuff, was seen in 7 patients (12.5\%), 5 (15\%) Aequalis and $2(8 \%)$ TESS recipients, but the difference was not significant (chi square test, $p r$ 0.472).
There were 5 patients $(8.9 \%)$ with posterior migration of the greater tuberosity, and none with superior migration. Interestingly, whenever the greater tuberosity was not depicted in the AP radiograms, it was demonstrated in axillary views and on CT, because it had migrated behind the prosthetic neck. There were no cases of migration of the lesser tuberosity. X-ray examination documented bony healing in 41 patients $(73.2 \%)$, malunion in $9(16.1 \%)$ and resorption in 6 patients $(10.7 \%)$.

Evaluation of the X-ray scans of Aequalis and TESS recipients showed tuberosity healing in $24(72 \%)$ and 17 patients (74\%); malunion in $5(15 \%)$ and 4 patients $(17.5 \%)$; and resorption in $4(12 \%)$ and $2(8 \%)$ patients, respectively (chi square test, $p r$ 0.908). Heterotopic ossification was seen in 5 patients $(8.9 \%)$ and was always related to a poor clinical outcome. Radiolucent lines were not observed when follow-up scans were compared with those taken immediately after the operation. Significantly greater Constant scores were found in patients with better radiographic findings, in terms of both bony healing (Kruskal-Wallis test, $p r$ 0.0001) and superior migration of the humeral head (Kruskal-Wallis test, pr 0.0001).

The patients who received a TESS implant were significantly younger than the Aequalis recipients (mean age 69.7 vs. 74 years) ( $t$ test, $p r 0.0413$ ) and had a significantly shorter mean follow-up (22.6 vs. 73.6 months, respectively) (Kruskal-Wallis test, $\mathrm{pr}$ 0.0001). There were no significant differences in involved side or fracture type (chi square test, $p r 0.938$ and 0.166 , respectively).

There were no neurological complications, wound infections or cases of implant instability.

\section{Discussion}

The optimal management of complex proximal humeral fractures is controversial. The main goals of treatment in these patients are a good functional result and pain relief. Neer was the first to advocate surgical treatment of three- and four-part fractures, due to the poor outcome of conservative management [15]. He treated three-part fractures by ORIF or HA and recommended total arthroplasty for four-part fractures, obtaining excellent or good results in at least $80 \%$ of

Table 1 Mean Constant scores of 56 patients at 12-98-month follow-up

\begin{tabular}{lcccc}
\hline Constant scores & All 56 patients (range) & 33 Aequalis patients (range) & 23 TESS patients (range) & Statistical test \\
\hline Adl & $16.3(12-20)$ & $16.2(12-20)$ & $16.6(12-20)$ & Kruskal-Wallis $p r 0.4761$ \\
Rom & $25.3(10-38)$ & $25.6(10-38)$ & $24.9(12-38)$ & Kruskal-Wallis $p r 0.5725$ \\
Pain & $14.0(10-15)$ & $14.1(10-15)$ & $13.9(10-15)$ & Chi square $p r 0.742$ \\
Strength & $3.3(0-8)$ & $3(0-8)$ & $3.7(2-8)$ & $t$ test $p r 0.1505$ \\
Total score & $59.2(38-76)$ & $59.3(39-75)$ & $59.1(38-75)$ & Kruskal-Wallis $p r 0.9335$ \\
\hline
\end{tabular}


patients. Zyto and co-workers [17] found no significant differences in Constant scores between patients with three- and four-part fractures. Compito et al. [18] reported excellent outcomes in $48.5 \%$ of patients. Kralinger et al. [19] described an Austrian multicentre study of 167 HA patients. At $>1$ year follow-up, healing of the tuberosities in anatomical position correlated with a better clinical outcome, $41.9 \%$ of patients being capable of active flexion $>90^{\circ}$. The older patients were at increased risk of pseudoarthrosis or malunion of the tuberosities. Outcomes appeared to be related to the experience of the individual centre. Kontakis et al. [20] reported the results of 28 procedures, where the Aequalis implant proved to be a safe and effective device. High rates of tuberosity healing were obtained, 24 patients being very satisfied or satisfied with the outcome and 18 achieving an active anterior elevation $>150^{\circ}$. At a mean follow-up of 39.3 months, the mean Constant score was 68.2.

Krishnan et al. [21] described $130 \mathrm{HA}$ procedures for fractures reconstructed with the Gothic arch technique. At an average 2 years of follow-up, anatomical tuberosity healing was achieved in $88 \%$, resulting in mean active anterior elevation of $129^{\circ}$ and a mean pain score of $1.2 / 10$.

Kontakis et al. reviewed 16 studies describing 810 HA procedures for acute proximal humeral fractures (nearly all four-part fractures and fracture-dislocations) with a mean follow-up duration of 3.7 years in 808 patients aged 67.7 years (range 22-91). Mean active anterior elevation was $105.7^{\circ}\left(10-180^{\circ}\right)$, mean abduction was $92.4^{\circ}$ $\left(15-170^{\circ}\right)$, and the mean Constant score was 56.63 (11-98). There were $11.15 \%$ complications related to fixation and healing of the tuberosities. Although most patients reported no or mild pain at the final follow-up, functional impairment was still considerable [14].

$\mathrm{HA}$ is indicated in patients who are medically stable, can tolerate extensive surgery, and can participate in postoperative rehabilitation. In young subjects with displaced proximal humeral fracture and in some patients with impacted four-part valgus fractures, ORIF may avoid prosthetic replacement and its potential complications [2226]. In such cases, even if bone necrosis or non-union does occur the tuberosities may be better positioned for conversion to a prosthesis [27]. Indications for HA include displaced three- and four-part fractures (according to Neer's classification), fracture-dislocations, head-splitting fractures not amenable to ORIF [14, 23, 24, 27-29], as well as severely osteoporotic bone, ORIF failure and bone necrosis. In addition, it is crucial to take into account the predictors of humeral head ischaemia [9]. Krishnan et al. [21] described four factors guiding in the choice of the treatment approach: age, bone quality, fracture pattern and timing of surgery. Although patients older than 70 years are candidates for arthroplasty [27, 30], chronological age is not an indication in itself, since patient activity level, the presence of osteoporosis and the fracture pattern are more important. HA is contraindicated in patients who cannot undergo surgery because they are medically unstable, in young, active patients, and in those with infection or axillary nerve palsy.

Tuberosity healing was the factor affecting outcome most significantly in our study; the Constant score was excellent in case of anatomical healing (Fig. 2) and poor in patients with malunion or resorption resulting in impaired ROM due to impingement (Fig. 3). Primary fixation of the tuberosities in a displaced position is associated with consistently poor results, emphasizing the value of using an image intensifier during the procedure, as also demonstrated in our study. A French multicentre study [31] of 406 patients showed that height of implantation, retroversion, tuberosity position, use of a fracture jig, rehabilitation and immobilization all have prognostic value.

Management of proximal humeral fractures with HA was very satisfactory or satisfactory in $91 \%$ of our patients. Accurate patient selection, careful surgical technique, and patient compliance with the rehabilitation programme seem to be critical factors in achieving good results. A mean Constant score of 59.2 reflects good joint, and ADL function with scarce or no pain in most patients.

Analysis of the clinical and radiographic results did not highlight significant differences between implant types. Use of the positioning guide for length and version with the Aequalis prosthesis was not found to be particularly effective, also given the complex procedure required to position the guide itself and the preoperative radiographic planning it involved. We feel the TESS system offers a number of advantages: humeral offset restoration thanks to its anatomical epiphysis, easy tuberosity fixation by passing sutures through the arches of the Corolla and improved tuberosity integration by virtue of the modest amount of metal and the possibility of adding growth factors.

Constant's score did not always match patient satisfaction, especially when the non-dominant limb was affected, since absence of pain encouraged acceptance of a mediocre range of motion.

Rehabilitation after prosthetic replacement for proximal humeral fractures remains contentious. Neer initially advocated early passive movement as the optimal postoperative protocol; Naranja and Iannotti [32] suggest aggressive rehabilitation, while Boileau et al. [33] recommend a more conservative protocol to avoid affecting tuberosity fixation. In a randomized controlled trial, Agorastides et al. [34] conclude that late mobilization after HA for proximal humeral fracture is as safe as early mobilization. Rehabilitation in our patients was influenced by socioeconomic status, since those who consistently followed their programme achieved better results, further confirming the central role of postoperative rehabilitation following shoulder surgery. 
Fig. 2 Female patient subjected to right HA for a four-part fracture at age 75 years. Followup X-rays at 40 months. Constant score 69. Patient very satisfied with outcome
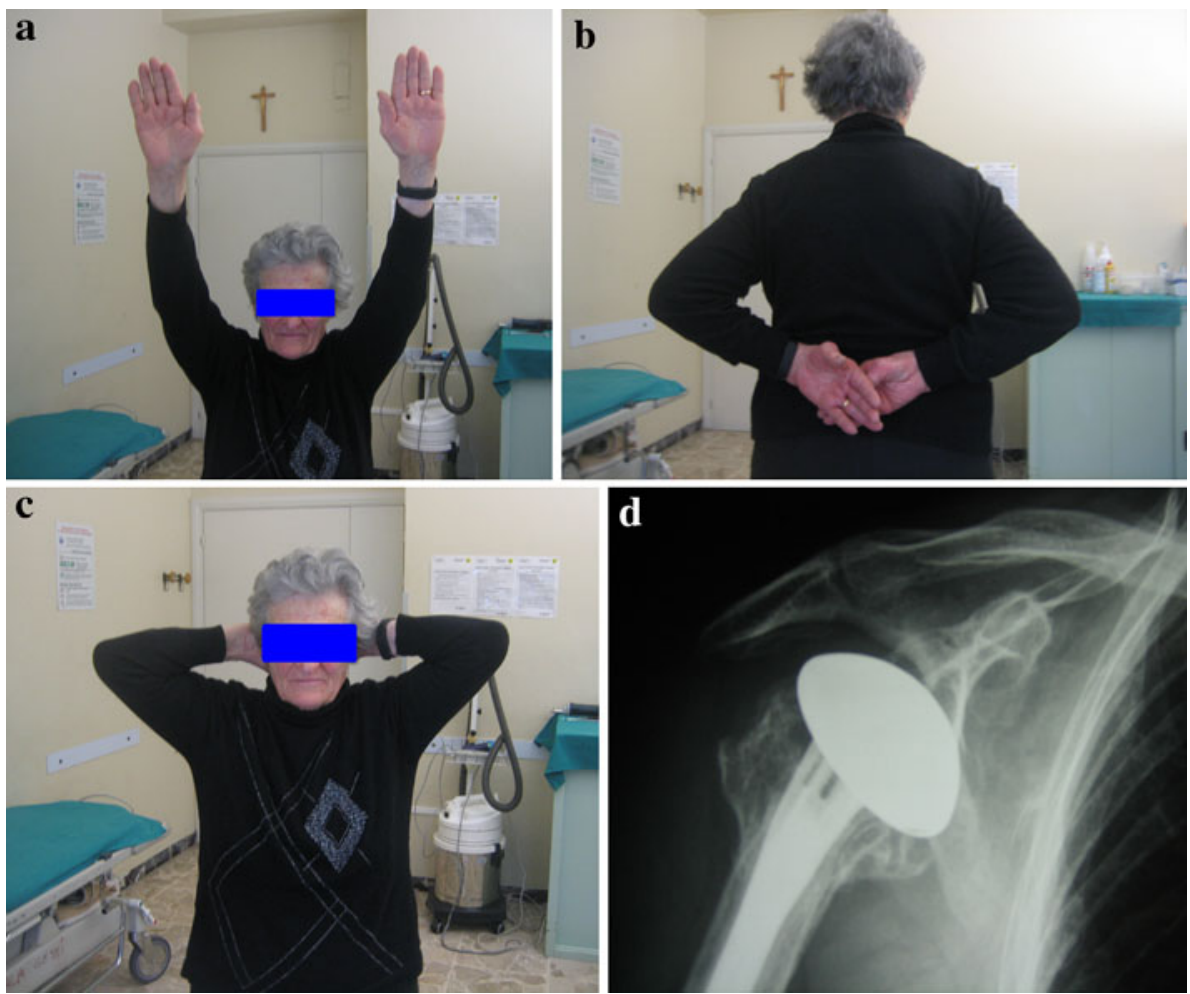
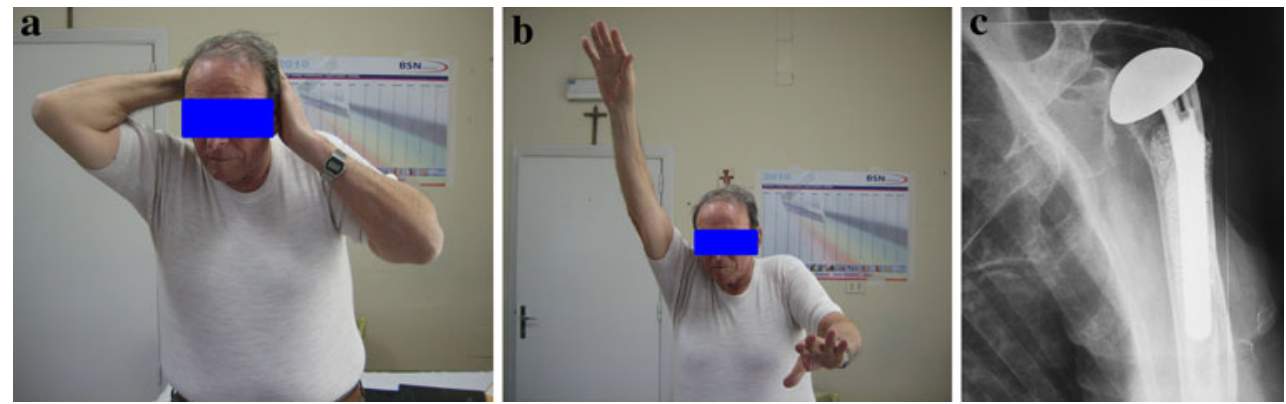

Fig. 3 Male patient operated on at age 75 years. Constant score 46. Follow-up X-rays at 6 years document tuberosity resorption with superior migration of the humeral head

Management of shoulder fractures by HA is technically demanding, as shown by the relationship between surgeon experience and outcome. A recent review [14] found that each surgeon performed 2.96 (range: 0.21-9.6) HA procedures a year for proximal humeral fractures. In our study all the operations were performed by the same surgeon, which may account for the high rate of anatomical tuberosity healing and the low rate of complications.

We consider restoration of proximal humerus geometry, anatomical reduction and stable reduction in the tuberosities, implant fixation and functional restoration of the rotator cuff to be critical factors in HA procedures.

Acknowledgments The authors are grateful to Drs. I. Panzini and E. Fabbri for statistical analysis and to Dr. S. Modena for language revision.
Conflict of interest The authors declare that there is no conflict of interest related to the publication of this article.

Open Access This article is distributed under the terms of the Creative Commons Attribution Noncommercial License which permits any noncommercial use, distribution, and reproduction in any medium, provided the original author(s) and source are credited.

\section{References}

1. Bengner U, Johnell O, Redlund-Johnell I (1988) Changes in the incidence of fractures of the upper end of the humerus during a 30 years study period: a study of 2,125 fractures. Clin Orthop 231:179-182

2. Lind T, Kroner TK, Jensen J (1989) The epidemiology of fractures of the proximal humerus. Arch Orthop Trauma Surg 108:285-287 
3. Horak J, Nilsson BE (1975) Epidemiology of fracture of the upper end of the humerus. Clin Orthop 112:250-253

4. Rose SH, Melton LJ III, Morrey BF, Ilstrup DM, Riggs BL (1982) Epidemiologic features of humeral fractures. Clin Orthop Relat Res 168:24-30

5. Moonot P, Ashwood N, Hamlet M (2007) Early results for treatment of three- and four-part fractures of the proximal humerus using the PHILOS plate system. J Bone Jt Surg [Br] 89:1206-1209

6. Gerber C, Werner CM, Vienne P (2004) Internal fixation of complex fractures of the proximal humerus. J Bone Jt Surg $[\mathrm{Br}]$ $86: 848-855$

7. Wijgman AJ, Roolker W, Patt TW, Raaymakers EL, Marti RK (2002) Open reduction and internal fixation of three and four-part fractures of the proximal part of the humerus. J Bone Jt Surg [Am] 84:1919-1925

8. Solberg BD, Moon CN, Franco DP, Paiement GD (2009) Surgical treatment of three and four-part proximal humeral fractures. J Bone Jt Surg [Am] 91(7):1689-1697

9. Hertel R, Hempfing A, Stiehler M, Leunig M (2004) Predictors of humeral head ischemia after intracapsular fracture of the proximal humerus. J Should Elbow Surg 13:427-433

10. Phipatanakul WP, Norris TR (2005) Indications for prosthetic replacement in proximal humeral fractures. Instr Course Lect 54:357-362

11. Mighell MA, Kolm GP, Collinge CA, Frankle MA (2003) Outcomes of hemiarthroplasty for fractures of the proximal humerus. J Should Elbow Surg 12:569-577

12. Bosch U, Skutek M, Fremery RW, Tscherne H (1998) Outcome after primary and secondary hemiarthroplasty in elderly patients with fractures of the proximal humerus. J Should Elbow Surg 7:479-484

13. Brian DS, Charles NM, Dennis PF, Guy DPM (2009) Surgical treatment of three and four-part proximal humeral fractures. J Bone Jt Surg [Am] 91:1689-1697

14. Kontakis G, Koutras C, Tosounidis T, Giannoudis P (2008) Early management of proximal humeral fractures with hemiarthroplasty: a systematic review. J Bone Jt Surg [Br] 90-B:1407-1413

15. Neer CS II (1970) Displaced proximal humeral fractures. II. Treatment of three- and four-part displacement. J Bone Jt Surg [Am] 52:1090-1103

16. Constant CR, Murley AH (1987) A clinical method of functional assessment of the shoulder. Clin Orthop Relat Res 214:160-164

17. Zyto K, Wallace WA, Frostick SP, Preston BJ (1998) Outcome after hemiarthroplasty for three- and four-part fractures of the proximal humerus. J Should Elbow Surg 7:85-89

18. Compito CA, Self EB, Bigliani LU (1994) Arthroplasty and acute shoulder trauma. Reasons for success and failure. Clin Orthop Relat Res 307:27-36

19. Kralinger F, Schwaiger R, Wambacher M, Farrell E, MenthChiari W, Lajtai G, Hübner C, Resch H (2004) Outcome after primary hemiarthroplasty for fracture of the head of the humerus. A retrospective multicentre study of 167 patients. J Bone Jt Surg [Br] 86:217-219
20. Kontakis GM, Tosounidis TI, Christoforakis Z, Hadjipavlou AG (2009) Early management of complex proximal humeral fractures using the Aequalis fracture prosthesis: a 2-5 years follow-up report. J Bone Jt Surg [Br] 91(10):1335-1340

21. Krishnan SG, Bennion PW, Reineck JR, Burkhead WZ (2008) Hemiarthroplasty for proximal humeral fracture: restoration of the Gothic arch. Orthop Clin North Am 39:441-450

22. Nho SJ, Brophy RH, Barker JU, Cornell CN, MacGillivray JD (2007) Innovations in the management of displaced proximal humerus fractures. J Am Acad Orthop Surg 15:12-26

23. DeFranco MJ, Brems JJ, Williams GR Jr, Iannotti JP (2006) Evaluation and management of valgus impacted four-part proximal humerus fractures. Clin Orthop Relat Res 442:109-114

24. Moeckel BH, Dines DM, Warren RF, Altchek DW (1992) Modular hemiarthroplasty for fractures of the proximal part of the humerus. J Bone Jt Surg [Am] 74:884-889

25. Ricchetti ET, DeMola PM, Roman D, Abboud JA (2009) The use of precontoured humeral locking plates in the management of displaced proximal humerus fracture. J Am Acad Orthop Surg 17:582-590

26. Thanasas C, Kontakis G, Angoules A, Limb D, Giannoudis P (2009) Treatment of proximal humerus fractures with locking plates: a systematic review. J Should Elbow Surg 18:837-844

27. Dines DM, Warren RF (2008) Arthroplasty for proximal humerus fractures. In: Dines DM, Lorich DG, Helfet DL (eds) Solutions for complex upper extremity trauma. Thieme, NY, pp 79-87

28. Bastian JD, Hertel R (2009) Osteosynthesis and hemiarthroplasty of fractures of the proximal humerus: outcomes in a consecutive case series. J Should Elbow Surg 18:216-219

29. Dines DM, Warren RF (1994) Modular shoulder hemiarthroplasty for acute fractures. Surgical considerations. Clin Orthop Relat Res 307:18-26

30. Hertel R (2005) Fractures of the proximal humerus in osteoporotic bone. Osteoporos Int 16(Suppl 2):S65-S72

31. Boileau P, Coste JS, Ahrens PM, Staccini P (2002) Prosthetic shoulder replacement for fracture: results of the multicenter study. In: Walch G, Boileau P, Mole D (eds) 2000 Shoulder prostheses: 2-10 years follow-up. Sauramps Medical, Montpellier, pp 561-573

32. Naranja RJ Jr, Iannotti JP (2000) Displaced three- and four-part proximal humerus fractures: evaluation and management. J Am Acad Orthop Surg 8:373-382

33. Boileau PCJ, Coste J-S, Ahrens PM, Staccini P (2001) Prosthetic shoulder replacement for fracture: results of the multicentre study. In: Walch G, Boileau P, Mole D (eds) 2000 Shoulder prostheses: 2-10 years follow up. Sauramps Medical, Montpellier, pp 561-578

34. Agorastides I, Sinopidis C, El Meligy M et al (2007) Early versus late mobilization after hemiarthroplasty for proximal humeral fractures. J Should Elbow Surg 16(3 Suppl):S33-S38 